\title{
Caracteristicas antropométricas, hábitos nutricionales, actividad física y consumo de alcohol en estudiantes universitarios
}

\author{
Anthropometric characteristics, nutritional habits, physical \\ activity and alcohol consumption in college students
}

Andrés-Felipe Villaquiran ${ }^{1}$; Paola Cuero ${ }^{1}$; Gineth-Magally Ceron ${ }^{2}$; Armando Ordoñez ${ }^{3}$; Sandra Jácome ${ }^{1}$

Forma de citar: Villaquiran AF, Cuero P, Ceron GM, Ordoñez A, Jácome S. Características antropométricas, hábitos nutricionales, actividad física y consumo de alcohol en estudiantes universitarios. Salud UIS. 2020; 52(2): 111-120. doi: http://dx.doi.org/10.18273/revsal.v52n2-2020005 (c) (1)

\section{Resumen}

Introducción: La vida universitaria trae consigo cambios en el estilo de vida, principalmente porque las conductas asumidas durante esta etapa son consideradas no saludables, situación preocupante para el desarrollo a temprana edad de enfermedades no transmisibles. Objetivo: Caracterizar los factores riesgo asociados al estilo de vida y a las enfermedades no transmisibles de los estudiantes de la facultad de ciencias contables, económicas y administrativas de una universidad pública del Cauca. Materiales y métodos: Estudio analítico de corte transversal, realizado a 99 estudiantes universitarios a quienes se les indagó por las características sociodemográficas, académicas, antropométricas y factores comportamentales asociados a enfermedades no transmisibles, basándose en los pasos 1 y 2 del instrumento STEPS validado por la Organización Mundial de la Salud. Resultados: Se encontró un promedio de edad de 22,5 años, un índice de masa corporal normal para ambos sexos y un índice cintura cadera que muestra mayor porcentaje de riesgo en el género femenino; se encontró un alto consumo de alcohol $(\mathrm{n}=60)$, los estudiantes consideraban tener un estilo de vida sedentario $(n=53)$, no fumaban $(n=88)$ y no consumían frutas y verduras $(\mathrm{n}=74)$, mientras que se encontró relación estadísticamente significativa entre sexo y motivos de diversión para consumir bebidas alcohólicas $(\mathrm{p}=0,039)$. Conclusión: Los hallazgos muestran una población universitaria vulnerable para sufrir de enfermedades no transmisibles debido a su percepción de vida sedentaria, la poca ingesta de frutas y verduras, el consumo de alcohol; conductas preocupantes que deben ser controladas a partir de propuestas de intervención que fomenten estilos de vida saludables.

Palabras clave: Enfermedad crónica; Factores de riesgo; Hábitos; Estilo de vida; Estudiantes.

1. Universidad del Cauca. Popayán.

2. Proyectamos: Consultorías y Servicios integrales SAS. Popayán, Cauca

3. Fundación Universitaria de Popayán. Cauca

Correspondencia a: Andrés Felipe Villaquiran Hurtado, Dirección: Calle 12 11ª-40 Las Américas, Popayán. Teléfono: +57 3104741014.

Correo electrónico: avillaquiran@unicauca.edu.co. 


\begin{abstract}
Introduction: College life carries changes in lifestyle mainly because the behaviors assumed during this stage are considered unhealthy, a worrying situation for early noncommunicable diseases development. Objective: To characterize the risk factors associated to lifestyle and noncommunicable diseases of the students from accounting, economic aculty and administrative sciences of a public university of Cauca. Materials and methods: A 99 university students cross-sectional, analytical study that asked about the sociodemographic, academic, anthropometric and behavioral factors associated with noncommunicable diseases, based on steps 1 and 2 of the STEPS instrument validated by the World Health Organization. Results: We found an average age of 22.5 years, a normal body mass index for both sexes and a hip waist index that shows a greater percentage of risk in the female gender, a high alcohol consumption was found, students considered having a sedentary lifestyle, did not smoke and did not consume fruits and vegetables, while a statistically significant relationship was found between sex and fun reasons to consume alcoholic beverages. Conclusion: The findings show a vulnerable university population to suffer noncommunicable diseases due to their perception of sedentary life, low intake of fruits and vegetables, alcohol consumption; Concerns that should be controlled based on intervention proposals that promote healthy lifestyles.
\end{abstract}

Keywords: Chronic disease; Risk factors; Habits; Lifestyle; Students.

\section{Introducción}

Las enfermedades no transmisibles (ENT) representan una sobrecarga de morbilidad y mortalidad considerablemente alta a nivel mundial ${ }^{1}$. El aumento de la prevalencia de estas patologías como la diabetes, el cáncer, las enfermedades cardiovasculares y pulmonares crónicas generan un impacto sobre la salud, los sistemas de atención, el costo de los servicios sanitarios y la disminución de los años de vida productiva de una persona ${ }^{2}$.

Las ENT causan la muerte de más de 41 millones de personas al año, siendo responsables del 85\% de las muertes prematuras, siendo el consumo de tabaco, el sedentarismo y las dietas inadecuadas los principales factores de riesgo asociados a las ENT ${ }^{3}$. Según el análisis de la situación de salud en Colombia para el 2018, de las atenciones prestadas por el sistema de salud el $65 \%$ fueron a personas con ENT. Adicionalmente, este estudio demostró que la juventud (14 a 26 años) colombiana acude a los servicios médicos principalmente por las ENT, situación que también se presentó en la edad adulta durante los años 2009 al $2018^{4}$.

De acuerdo a lo anterior, reducir la carga de estas enfermedades parece ser uno de los retos más importantes durante los próximos años para garantizar el desarrollo sostenible de la región ${ }^{3}$. Las ENT son trastornos que reducen el capital humano, disminuyen el tiempo laboral del individuo, reducen los ingresos y generan un retraso en el desarrollo económico de los territorios, convirtiéndose en uno de los fenómenos más costosos y persistentes para la población de América Latina 5 .

La vida universitaria supone una etapa crítica para el joven estudiante, la influencia de los factores emocionales, fisiológicos, sociales, económicos y culturales generan cambios sustanciales en el estilo de $v^{2} a^{6}$, produciendo modificaciones que impactan negativamente los hábitos nutricionales, aumentando el consumo de alcohol y cigarrillo, y disminuyendo los niveles de actividad física ${ }^{7,8}$. Las largas jornadas de clases, los múltiples deberes estudiantiles, el estrés que genera el rendir académicamente, las creencias, las tradiciones, las variaciones en el estado de ánimo y los medios de comunicación son algunos de los factores que pueden traer consigo conductas poco saludables durante la etapa universitaria en los estudiantes de ciencias Contables, Económicas y Administrativas9. Al respecto, existe una alta prevalencia de sedentarismo en los estudiantes universitarios, siendo menor en carreras afines al movimiento. Aunque, se ha reportado que los estudiantes de Contaduría Pública son físicamente activos, otros programas afines, como administración de negocios y de comercio presentan niveles muy bajos de actividad física entre sus estudiantes ${ }^{10}$. Estas conductas "mal sanas" pueden mantenerse durante la vida laboral, un estudio realizado en trabajadores administrativos del sector publico muestra que el $67 \%$ de los trabajadores no realiza ningún tipo de actividad física y el $54 \%$ de los encuestados permanece más de siete horas sentados frente a un ordenador ${ }^{11}$. 
Respecto al sedentarismo y la importancia de recomendar actividad física, las enfermedades cardiovasculares aumentan significativamente al permanecer sentados de 6 a 8 horas diarias o de 3 a 4 horas al día viendo televisión ${ }^{12}$. Por su parte, la actividad física puede traer beneficios y control sobre factores de riesgo cardiovascular como lo son: el colesterol, los triglicéridos, la obesidad, la hipertensión, el estrés y el consumo de tabaco y alcohol, lo que representa un efecto protector sobre la salud del individuo ${ }^{13}$.

De acuerdo a lo anterior, la evaluación de los diferentes factores de riesgo para padecer ENT debe ser un pilar fundamental para la vigilancia y monitorización de estas enfermedades. Esta evaluación debe reunir, analizar y distribuir la información de manera sencilla y estandarizada, pero a la vez debe ser flexible para incorporar variables relacionadas al contexto donde se realice la valoración. Dentro de los criterios para evaluar los factores de riesgo, la Organización Mundial de la Salud (OMS) propone tener en cuenta en la estimación el consumo de tabaco, la ingesta de alcohol, el estado nutricional y los niveles de actividad física, así como también la toma de medidas antropométricas y el análisis bioquímico a través de muestras de sangre. La OMS recoge esta propuesta en su método de vigilancia STEPwise, el cual busca simplificar por medio de una herramienta sencilla la valoración de los factores asociados con las $\mathrm{ENT}^{14}$. La implementación de este instrumento en población universitaria puede ser una opción viable para conocer y entender el fenómeno de la ENT en la población joven, lo que permitirá tomar las medidas de prevención primaria y secundaria para proteger la salud de los estudiantes y contribuir más adelante con programas de promoción de la salud específicos para la población estudiantil.

Conociendo el impacto a nivel mundial que generan las ENT, la importancia que tiene un estilo de vida saludable como factor protector, entendiendo la vulnerabilidad que supone la universidad en el cambio de los procesos de autocuidado y al mismo tiempo encontrar pocas investigaciones que identifiquen los factores de riesgo para ENT en estudiantes universitarios de ciencias contables, económicas y administrativas de la región. El objetivo de este estudio fue caracterizar los factores de riesgo para ENT en estudiantes universitarios de ciencias contables, económicas y administrativas de una universidad pública del departamento del cauca.

\section{Materiales y métodos}

Estudio analítico de corte transversal, realizado en estudiantes universitarios de la Facultad de Ciencias Contables, Económicas y Administrativas de una Universidad pública en el departamento del Cauca en el segundo periodo de 2016.

Para la participación en el estudio se tuvieron en cuenta como criterios de inclusión estar matriculados $\mathrm{y}$ activos en el registro académico universitario en el segundo periodo de 2016, ser estudiante de los programas académicos ofertados por la facultad, manifestar su deseo voluntario de colaboración, firmar el consentimiento informado y diligenciar completamente la encuesta. Respecto al cálculo del tamaño de la muestra se utilizó el programa EPIDAT versión 3.1 , con un tamaño de población de 1.581 estudiantes con una prevalencia del $16,63 \%$, un nivel de confianza del $95 \%$ y precisión de 7,5\%, obteniendo como mínimo una muestra de 90 estudiantes, para evitar pérdidas se tomó un total de 99 universitarios de los programas académicos de turismo, contaduría y administración de empresas. La selección de los participantes se realizó mediante un muestreo aleatorizado simple a partir de las listas entregadas por el sistema de registro académico de la Universidad donde se encontraban codificados los estudiantes según programa y semestre. Los participantes fueron contactados mediante búsqueda activa de acuerdo a sus horarios establecidos para el diligenciamiento de la encuesta.

A cada participante se le explicó el objetivo del estudio y la metodología de la investigación, se le respondían las dudas y preguntas que pudiesen tener y se procedía a la firma del consentimiento informado. El diligenciamiento de la encuesta fue realizado de manera individual, en ella se preguntaba por las características sociodemográficas, académicas y factores de riesgo para ENT basándose en los pasos 1 y 2 del instrumento STEPS validado por la Organización Mundial de la Salud ${ }^{14}$. En las mediciones del paso 1 se tuvieron en cuenta los ítems relacionados con el consumo de cigarrillo, alcohol, dieta, tensión arterial elevada, medidas físicas y actividad física. Para el paso 2 se tuvieron en cuenta: la estatura, el peso, el perímetro de cintura y cadera. Al cuestionario se le incluyeron preguntas relacionadas con el ámbito académico (semestre, carrera, promedio de carrera, repetición de asignaturas y ocupación adicional), con 
el objetivo de analizar si existía relación entre los factores de riesgo para enfermedades no trasmisibles y el rendimiento académico. Para las toma de las medidas antropométricas como talla, peso, índice de masa corporal, perímetro de cintura y cadera se tuvieron en cuenta las recomendaciones de la International Society for the Advancement of Kineanthropometry (ISAK) ${ }^{15}$. Además, Los evaluadores fueron entrenados para la toma de medidas antropométricas de tal manera que en la prueba piloto sus mediciones dieran iguales en tres tomas repetidas al mismo sujeto. Adicionalmente, al instrumento utilizado se le realizó aseguramiento de la validez mediante prueba piloto realizada a 30 sujetos con características similares a la muestra del estudio, encontrándose que las preguntas planteadas fueron entendidas y respondidas por los participantes.

Para el procesamiento de la información se empleó el programa PSPP y R Wizard, software de uso libre. Para la interpretación de los datos se realizó un análisis descriptivo con distribuciones de frecuencia y
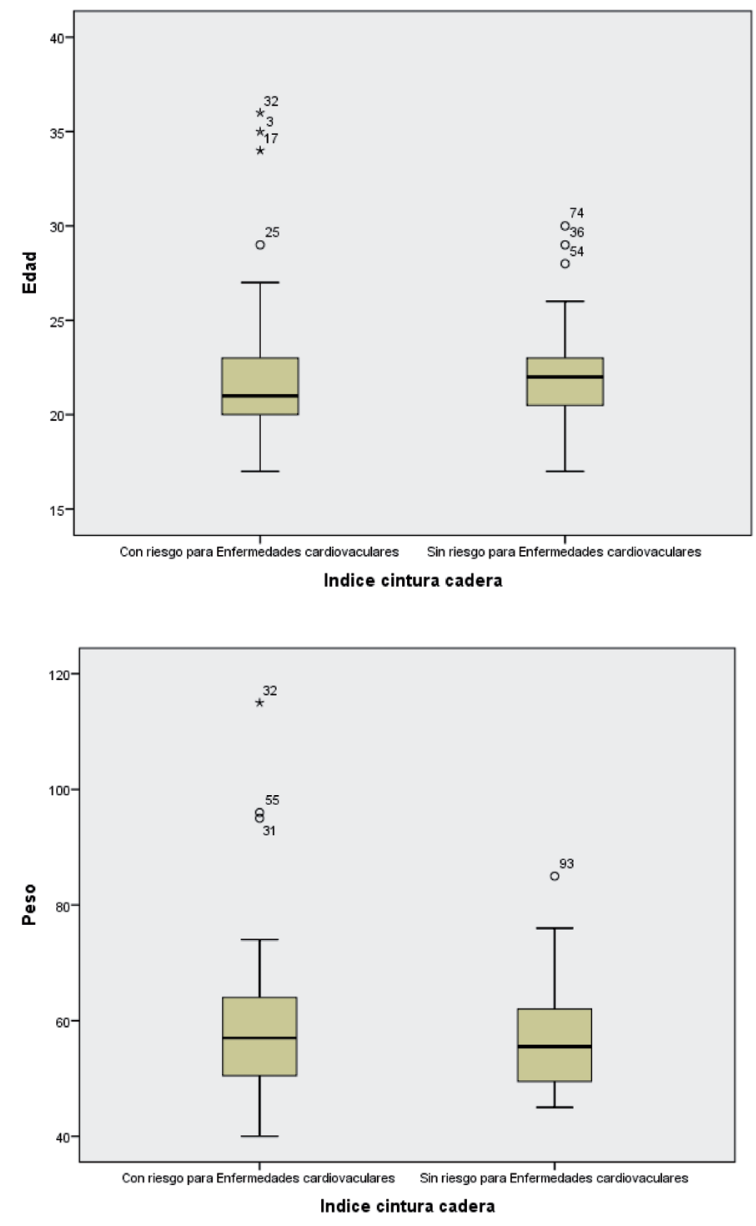

porcentaje, posteriormente un análisis inferencial para lo cual se utilizaron las pruebas no paramétricas $U$ de Mann Whitney y chi cuadrado, se realizaron pruebas de correlación, con cálculo del OR. Todas las pruebas se tomaron con un nivel de confianza del $95 \%$.

Para la realización de la investigación se tuvo en cuenta los aspectos éticos de la declaración de HELSINKI ${ }^{16}$ y se contó con el aval del Comité de ética de la Universidad del Cauca.

\section{Resultados}

Se contó con la participación de 99 estudiantes de los programas de administración de empresas, contaduría y turismo. Se observó que la mayoría de población tiene 20 años, con un promedio de 22,5 33,526 años, encontrándose un promedio de edad más elevada para el grupo de ICC sin riesgo. La talla en la población tiene un promedio de $161,76 \pm 7,263 \mathrm{~cm}$. El peso tiene un promedio de 58,58 $\pm 11,892$ kilos (Figura 1).

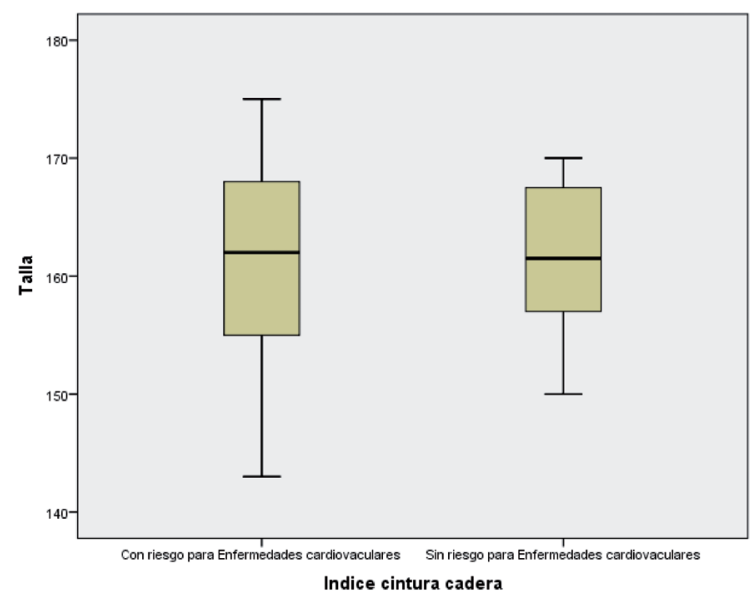

\begin{tabular}{lrrr}
\hline & Edad & \multicolumn{1}{c}{ Peso } & \multicolumn{1}{c}{ Talla } \\
\hline Media & 22,05 & 58,58 & 161,76 \\
Desviación estándar & 3,526 & 11,892 & 7,263 \\
Mínimo & 17 & 40 & 143 \\
Máximo & 36 & 115 & 175 \\
\hline
\end{tabular}

Figura 1. Distribución de edad, talla y peso según índice cintura cadera (ICC) y estadísticos descriptivos para edad, peso y talla. Fuente: Grupo investigador. 
La población en su mayoría para ambos sexos tiene procedencia urbana y estado civil soltero. A pesar que quienes sólo estudian tienen mayor porcentaje para ambos sexos, se observa comparativamente un mayor porcentaje de estudiantes que si trabajan en el género masculino. El régimen de salud tanto en hombres como en mujeres es en su mayoría subsidiado, el estrato socioeconómico de la población el mayor porcentaje se encuentra en bajo. Respecto a las características académicas, es mayor el porcentaje de estudiantes con promedio entre 3,5 y 3,9. En los antecedentes patológicos, se encuentran los antecedentes familiares para diabetes más frecuente en el género femenino, al igual que cáncer, enfermedades respiratorias, enfermedades del corazón, hipertensión arterial, ECV y lípidos y colesterol alto. Respecto a antecedentes personales, se encontró antecedentes patológicos de enfermedad respiratoria mayor en el género masculino, antecedentes patológicos personales y familiares para enfermedad respiratoria y para hipertensión arterial en el género masculino y para enfermedades del corazón en ambos sexos. El IMC se encuentra mayor frecuencia catalogado como normopeso y el ICC muestra mayor porcentaje de riesgo en el género femenino (Tabla 1).

Se observó que de los estudiantes de los tres programas que realizan actividad física, la mayor frecuencia la presentan los estudiantes de administración de empresas que se encuentran en sus primeros cinco semestres, sin embargo, contradictoriamente este mismo grupo presenta la mayor frecuencia de considerar tener un estilo de vida sedentario. Por otra parte, los hábitos de vida no saludables se encuentran con mayor frecuencia el consumo de alcohol en el mismo grupo de administración de los primeros cinco semestres al igual que en el grupo de Turismo en los semestres a partir del quinto, en estos dos grupos también se encuentra la mayor frecuencia de consumo de alcohol hasta la embriaguez. El hábito de fumar presenta su mayor frecuencia en estudiantes de turismo en los semestres de quinto en adelante, al igual que el ser fumador pasivo. (Tabla 2.)

\section{Discusión}

Las condiciones particulares a las cuales son sometidos los estudiantes universitarios generan cambios en el estilo de vida, trayendo implicaciones negativas para los hábitos alimenticios, actividad física, consumo de alcohol y tabaco contribuyendo con la aparición de factores de riesgo cardiovascular durante su etapa de formación universitaria ${ }^{17}$.
Tabla 1. Características sociodemográficas, académicas y clínicas de los participantes según sexo.

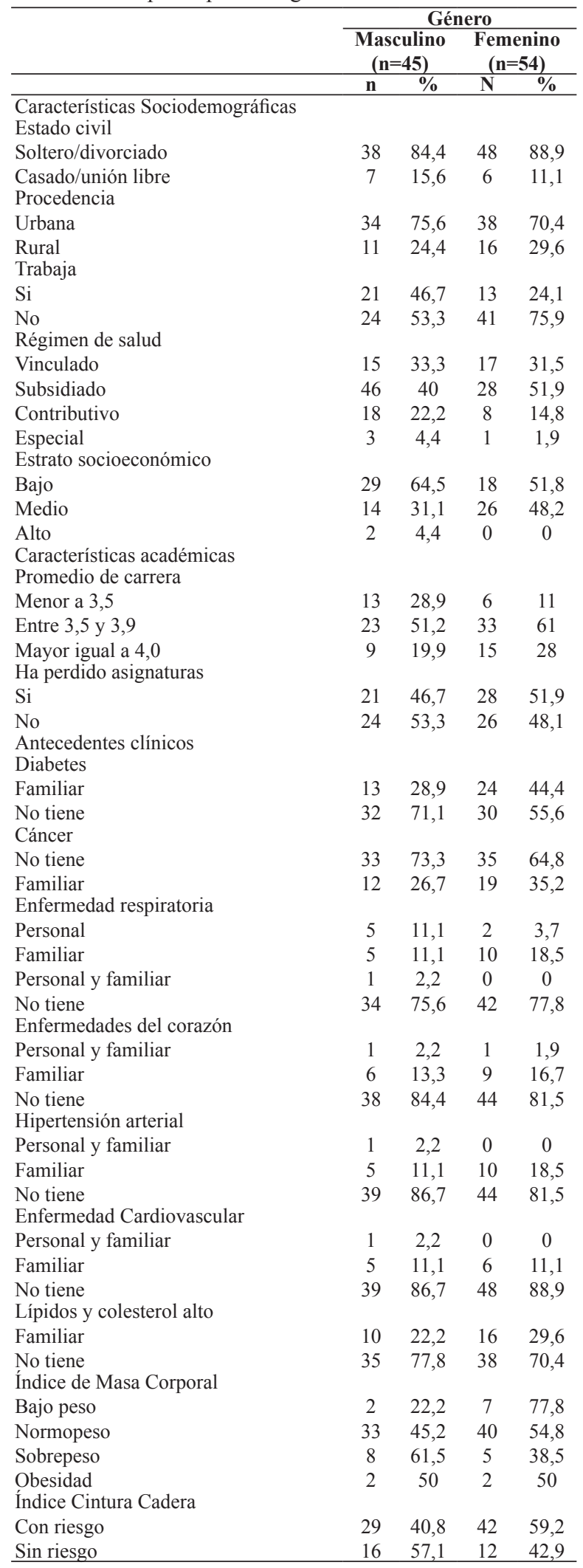

Fuente: Grupo investigador. 
Tabla 2. Distribución de hábitos de vida según de carrera y semestre

\begin{tabular}{|c|c|c|c|c|c|c|c|c|c|c|c|c|c|}
\hline & \multirow[b]{2}{*}{ Semestre } & \multicolumn{2}{|c|}{$\begin{array}{l}\text { Realiza alguna } \\
\text { actividad física }\end{array}$} & \multicolumn{2}{|c|}{$\begin{array}{c}\text { Considera } \\
\text { que tiene un } \\
\text { estilo de vida } \\
\text { sedentario }\end{array}$} & \multicolumn{2}{|c|}{$\begin{array}{l}\text { Consume } \\
\text { alcohol }\end{array}$} & \multicolumn{2}{|c|}{$\begin{array}{c}\text { Consume } \\
\text { alcohol } \\
\text { hasta la } \\
\text { embriaguez }\end{array}$} & \multicolumn{2}{|c|}{ Fuma } & \multicolumn{2}{|c|}{$\begin{array}{c}\text { Se } \\
\text { considera } \\
\text { fumador } \\
\text { pasivo }\end{array}$} \\
\hline & & Si & No & Si & No & Si & No & Si & No & Si & No & Si & No \\
\hline \multirow{2}{*}{ Contaduría } & Hasta quinto & 13 & 5 & 10 & 8 & 7 & 11 & 3 & 15 & 1 & 17 & 4 & 14 \\
\hline & De quinto en adelante & 7 & 8 & 8 & 7 & 9 & 6 & 1 & 14 & 1 & 14 & 1 & 14 \\
\hline \multirow{2}{*}{ Administración } & Hasta quinto & 18 & 6 & 13 & 11 & 16 & 8 & 6 & 18 & 1 & 23 & 2 & 22 \\
\hline & De quinto en adelante & 10 & 6 & 9 & 7 & 12 & 4 & 3 & 13 & 3 & 13 & 5 & 11 \\
\hline \multirow{2}{*}{ Turismo } & Hasta quinto & 2 & 2 & 1 & 3 & 0 & 4 & 0 & 4 & 0 & 4 & 2 & 2 \\
\hline & De quinto en adelante & 15 & 7 & 12 & 10 & 16 & 6 & 6 & 16 & 5 & 17 & 9 & 13 \\
\hline Total & & 65 & 34 & 53 & 46 & 60 & 39 & 19 & 80 & 11 & 88 & 23 & 76 \\
\hline
\end{tabular}

Fuente: Grupo investigador.

En relación con las medidas antropométricas se encontró que la mayoría de estudiantes presento un índice de masa corporal (IMC) normal, resultados similares a los reportados por El-Hayek, et al. en estudiantes de una universidad privada en Lebanon ${ }^{18}$. El manejo de una dieta saludable ayuda en el control de está variable, estructuras dietarías basadas en grasas, carnes rojas, patatas y azúcar se asocian a perfiles altos de IMC en estudiantes universitarios ${ }^{19}$. Mientras tanto, el índice cintura cadera (ICC) arrojó un mayor riesgo de sufrir enfermedades cardiovasculares, situación que debe ser tenida en cuenta para la prevención y control de la obesidad que conlleva a un alto riesgo de sufrir enfermedades crónicas ${ }^{18}$. Por su parte la obesidad ha sido relacionada como un factor de riesgo importante en la aparición temprana de enfermedades cardiovasculares; sobre esto, Nkeh, et al. encontraron que incrementos en la circunferencia de la cintura y cadera se asociaron con presencia de hipertensión y pre hipertensión en mujeres universitarias ${ }^{20}$.

Una evaluación y un control temprano de estos factores antropométricos pueden prevenir el riesgo de ateroesclerosis y dislipidemias asociados a enfermedades cardiovasculares ${ }^{21}$. Estos incrementos desfavorables en el IMC y el ICC pueden generarse debido a los múltiples cambios en el estilo de vida del universitario como alejarse del núcleo familiar, asumir la responsabilidad de su alimentación, la vida social nocturna, las ocupaciones académicas, la situación económica y los cambios emocionales pueden favorecer conductas nutricionales negativas y poco asertivas que alteran los parámetros antropométricos de la población universitaria ${ }^{22}$.
La actividad física ha sido relacionada con la prevención y protección de la salud cardiovascular, también se ha establecido la importancia que presenta en la capacidad cognitiva de adolescentes y jóvenes ${ }^{23}$. Los resultados encontrados en este estudio sugieren que la población universitaria participante realiza actividad física en un alto porcentaje, datos que difieren con lo reportado en estudiantes universitarios chilenos, los cuales presentaban altos niveles de inactividad física producto de la falta de tiempo y la pereza que les supone ser $\operatorname{activos}^{24,25}$. Por su parte, en un estudio realizado en estudiantes universitarios chilenos se encontró que factores como el consumo de tabaco, antecedentes de lesión musculo- esquelética, tener sobrepeso, consumir comida chatarra y estudiar durante muchas horas se asocian a un bajo nivel de actividad física ${ }^{26}$. Sobre esto, bajos niveles de actividad física se han relacionado con mayor probabilidad de tener obesidad abdominal, síndrome metabólico y triglicéridos altos ${ }^{27}$. Es importante tener en cuenta que los motivos más frecuentes para realizar actividad física por parte de los estudiantes universitarios hombres están relacionados con la diversión y la competición, mientras que las mujeres se ven motivadas por el mantenimiento de la salud y la estética corporal ${ }^{28}$. Si bien es cierto que los aspectos motivacionales juegan un papel importante para la práctica de ejercicio, las universidades pueden contribuir a mejorar los indicadores en los niveles de actividad física de sus estudiantes, optimizando los procesos de información utilizados para promocionar los programas deportivos, disminuyendo el costo en las ofertas deportivas, permitiendo una mayor flexibilidad en los planes de estudio para incluir espacios para hacer deporte y la promoción del uso de la bicicleta; 
estrategias que pueden contribuir en la protección de la salud de los universitarios ${ }^{29}$.

Con respecto a las conductas no saludables como el consumo de alcohol, se encontró una alta ingesta en los universitarios, resultados muy similares a los reportados por Maphisa y Young en estudiantes Sur-africanos, donde encontraron una prevalencia del $68,5 \%$ para la ingesta de bebidas alcohólicas, siendo los motivos más frecuentes para hacerlo las motivaciones sociales en los hombres y circunstancias ligadas al afrontamiento en las mujeres universitarias ${ }^{30}$. Por su parte Atwell, et al. sugieren intervenciones enfocadas en el aprendizaje de beber moderadamente, cambiar las percepciones sobre el alcohol como facilitador de la interacción social y la experimentación de nuevas sensaciones ${ }^{31}$.

El consumo de alcohol hasta la embriaguez y la dificultad para rechazar invitaciones para beber licor, son los principales factores para realizar esta conducta "mal sana". Al respecto, Tembo, et al. afirman que estudiantes universitarios entre los 18 y 24 años, quienes consumen alcohol hasta la embriaguez tienen 1,2 veces más probabilidades de sufrir problemas psicológicos, llegar tarde y/o faltar a clase y presentar mayor incapacidad para concentrarse. Por estas razones, también se sugieren intervenciones con estrategias múltiples educativas, ambientales y económicas que reduzcan los posibles daños a la salud la ingesta desmesurada de licor durante la estancia universitaria ${ }^{32}$.

Si bien el fumar no es un hábito frecuente para la población de estudio, Peltzer y Pengpid encontraron una relación concurrente entre el hábito de fumar y el consumo de alcohol; conductas asociadas a un aumento de los problemas de salud, deterioro mental, problemas físicos y uso de sustancias ilegales ${ }^{33}$. Por otro lado, un estudio realizado por Saridi, et al. concluyó que los estudiantes universitarios creen que la implementación de campañas y programas de educación son mal diseñados y poco promovidos por parte de las autoridades, además que las leyes para prohibir el fumar en lugares cerrados puede disminuir el consumo, aunque estudiantes no fumadores en un $34 \%$ han sido expuestos en ambientes cerrados a humo secundario ${ }^{34}$.

Otro de los factores de riesgo para tener en cuenta son los hábitos nutricionales, la poca ingesta de frutas y verduras, proteínas y lácteos sumado a dietas ricas en carbohidratos son los datos más relevantes encontrados en los participantes de esta investigación, comportamientos que se catalogan como inadecuados. Asimismo, estudiantes argentinos entre los 23 a 33 años mostraron patrones alimentarios deficientes presentando asociación significativa con presencia de sobrepeso y aumento de la grasa corporal $^{35}$. El género, la edad, el año de estudio, la ubicación geográfica y la capacidad para cocinar son algunos de los factores que pueden incidir en los patrones dietarios de los estudiantes, estos esquemas cuando son menos favorables están asociados a un mayor consumo de cigarrillo, baja actividad física, comportamientos nada beneficiosos con el estilo de vida $^{36}$. Adicionalmente, Valladares encontró asociación entre el comportamiento alimentario y el rendimiento académico en estudiantes mujeres en Chile $^{37}$, esto sugiere que las acciones de educación nutricional para esta población podrían contribuir con el estado alimentario y la salud, entendiendo que las conductas alimentarias constituyen un factor potencialmente modificable y determinante en el estilo de vida saludable ${ }^{38}$.

Tabla 3. Frecuencia de hábitos alimentarios según de carrera y semestre

\begin{tabular}{|c|c|c|c|c|c|c|c|c|c|c|c|}
\hline & \multirow[b]{2}{*}{ Semestre } & \multicolumn{2}{|c|}{ Frutas y Verduras } & \multicolumn{2}{|c|}{ Carbohidratos } & \multicolumn{2}{|c|}{ Grasas } & \multicolumn{2}{|c|}{ Proteína } & \multicolumn{2}{|c|}{ Productos lácteos } \\
\hline & & $\mathbf{S i}$ & No & $\mathbf{S i}$ & No & $\mathbf{S i}$ & No & $\mathbf{S i}$ & No & $\mathbf{S i}$ & No \\
\hline \multirow{2}{*}{ Contaduría } & Hasta quinto & 5 & 13 & 10 & 8 & 5 & 13 & 6 & 12 & 2 & 16 \\
\hline & De quinto en adelante & 2 & 13 & 8 & 7 & 7 & 8 & 1 & 14 & 1 & 14 \\
\hline \multirow{2}{*}{ Administración } & Hasta quinto & 5 & 19 & 16 & 8 & 5 & 19 & 10 & 14 & 2 & 22 \\
\hline & De quinto en adelante & 2 & 14 & 13 & 3 & 6 & 10 & 6 & 10 & 3 & 13 \\
\hline \multirow{2}{*}{ Turismo } & Hasta quinto & 0 & 4 & 4 & 0 & 1 & 3 & 0 & 4 & 0 & 4 \\
\hline & De quinto en adelante & 11 & 11 & 15 & 7 & 6 & 16 & 11 & 11 & 5 & 17 \\
\hline Total & & 25 & 74 & 66 & 33 & 30 & 69 & 34 & 65 & 13 & 86 \\
\hline
\end{tabular}

Fuente: Grupo investigador. 
Tabla 4. Relación entre género, ocupación y estilo de vida

\begin{tabular}{|c|c|c|c|c|c|c|c|}
\hline & \multicolumn{4}{|c|}{ Género } & \multirow{3}{*}{$\mathbf{P}$} & \multirow{3}{*}{ OR } & \multirow{3}{*}{ IC95\% } \\
\hline & \multicolumn{2}{|c|}{ Masculino } & \multicolumn{2}{|c|}{ Femenino } & & & \\
\hline & $\mathbf{n}$ & $\%$ & $\mathbf{n}$ & $\%$ & & & \\
\hline \multicolumn{8}{|l|}{ Ocupación } \\
\hline Estudia y trabaja & 21 & 21,2 & 13 & 13,1 & 0,018 & 2,76 & $1,173-6,493$ \\
\hline Sólo estudia & 24 & 24,2 & 41 & 41,4 & & & \\
\hline \multicolumn{8}{|c|}{$\begin{array}{l}\text { Razones que lo llevan a } \\
\text { consumir bebidas alcohólicas }\end{array}$} \\
\hline Diversión & 29 & 29,3 & 23 & 23,3 & & & \\
\hline Soledad & 1 & 1 & 1 & 1 & & & \\
\hline Carga académica & 2 & 2 & 1 & 1 & 0,039 & & \\
\hline Otro & 1 & 1 & 7 & 7,1 & & & \\
\hline No consume & 12 & 12,1 & 22 & 22,2 & & & \\
\hline \multicolumn{8}{|l|}{ Sedentarismo } \\
\hline $\mathrm{Si}$ & 7 & 7,1 & 27 & 27,3 & 0,000 & 0,184 & $0,070-0,484$ \\
\hline No & 38 & 38,4 & 27 & 27,3 & & & \\
\hline \multicolumn{8}{|l|}{ Trotar } \\
\hline $\mathrm{Si}$ & 8 & 8,1 & 1 & 1 & 0,006 & & \\
\hline No & 37 & 37,4 & 53 & 53,5 & & & \\
\hline \multicolumn{8}{|l|}{ Montar bicicleta } \\
\hline $\mathrm{Si}$ & 14 & 14,1 & 6 & 6,1 & 0,014 & & \\
\hline No & 31 & 31,3 & 48 & 48,5 & & & \\
\hline \multicolumn{8}{|l|}{$\begin{array}{l}\text { Razones de no realizar } \\
\text { actividad física }\end{array}$} \\
\hline Falta de tiempo & 3 & 3 & 10 & 10,1 & & & \\
\hline Condición física & 1 & 1 & 1 & 1 & 0,001 & & \\
\hline Falta de interés & 3 & 3 & 15 & 15,2 & & & \\
\hline Otro & 38 & 38,4 & 28 & 28,3 & & & \\
\hline \multicolumn{8}{|c|}{$\begin{array}{l}\text { Consumo de alcohol hasta } \\
\text { la embriaguez }\end{array}$} \\
\hline $\mathrm{Si}$ & 16 & 16,2 & 3 & 3 & & & $2,519-34,924$ \\
\hline No & 29 & 29,3 & 51 & 51,5 & 0,000 & 9,379 & \\
\hline \multicolumn{8}{|c|}{$\begin{array}{l}\text { Le resulta difícil decir que no a } \\
\text { una invitación a tomar bebidas } \\
\text { alcohólicas }\end{array}$} \\
\hline $\mathrm{Si}$ & 11 & 11,1 & 2 & 2 & & & $1,755-40,328$ \\
\hline No & 34 & 34,3 & 52 & 52,5 & 0,002 & 8,412 & \\
\hline \multicolumn{8}{|c|}{ Frecuencia consumo bebidas alcohólicas } \\
\hline Todos fines de semana & 8 & 8,1 & 1 & 1 & & & \\
\hline $1 \mathrm{vez}$ al mes & 9 & 9,1 & 7 & 7,1 & 0,02 & & \\
\hline Rara vez & 17 & 17,2 & 24 & 24,2 & & & \\
\hline Nunca & 11 & 11,1 & 22 & 22,2 & & & \\
\hline
\end{tabular}

Fuente: Grupo investigador.

\section{Conclusión}

La población estudiantil universitaria presenta conductas no saludables que pueden aumentar el riesgo de enfermedades no transmisibles a temprana edad, el peligro de obesidad y diabetes producto de una dieta no saludable y la alarma que debe generar la percepción de un estilo de vida sedentario con poca actividad física durante el tiempo libre para sufrir enfermedades cardiovasculares, deben ser suficientes motivos para iniciar programas educativos y de intervención que fomenten estilos de vida saludables que vayan a favor de la salud y el bienestar del estudiante desde una perspectiva de formación integral.

\section{Agradecimientos}

Agradecemos a los estudiantes auxiliares de investigación por su ayuda en la recolección de datos, al programa de fisioterapia de la Universidad del Cauca por su compromiso con la investigación. 


\section{Conflicto de intereses}

Los autores declaramos no tener ningún conflicto de intereses.

\section{Referencias}

1. Menon V, Edathadathil F, Sathyapalan D, Moni M, Don A, Balachandran S, et al. Assessment of 2013 AHA/ACC ASCVD risk scores with behavioral characteristics of an urban cohort in India. Medicine (Baltimore). 2016; 95(49): e5542. doi: 10.1097/ MD.0000000000005542.

2. Uribe Caballero CV, Alonso Palacio LM. Noncommunicable chronic diseases. Its time to think about them. Rev Salud Uninorte. 2010; 26(2): 7-9.

3. Organización Mundial de la Salud. Enfermedades no transmisibles. 2018; https://www.who.int/es/ news-room/fact sheets/detail/noncommunicablediseases.

4. Dirección de epidemiología y demografíaMinisterio de salud y protección social. analisis de situación de salud. Colombia, 2018. Imprenta Nacional de Colombia. 2019: 1-273.

5. Organización Panamericana de la Salud y la Universidad de Washington. Las dimensiones economicas de las enfermedades no transmisibles en América Latina y el Caribe. 2017: 1-188.

6. Cervera Burriel F, Serrano Urrea R, García C, Milla Tobarra M, García Meseguer MJ. Hábitos alimentarios y evaluación nutricional en una población universitaria. Nutr Hosp. 2013; 28(2): 438-446. doi: http://dx.doi.org/10.3305/ nh.2013.28.2.6303.

7. Pulido-Medina C, Reyes Suarez N, León Aristizabal A, Cárdenas Casallas J, Rivera Samian S, Rodríguez Saenz AY. Factores de riesgo de enfermedades crónicas no transmisibles en estudiantesde ciencias basicas de la Escuela de Medicina de la Universidad Pedagogica y Tecnologica de Colombia. Rev salud Hist Sanid. 2015; 10(1): 15-25.

8. Villarroel A, Saravia A, Castillo R. Estilos de vida y su relación con las caracteristicas en estudiantes de la facultad de enfermeria de la Universidad Nacional San Luis Gonzaga de ICA 2015. Rev Enferm Vanguard. 2017; 5(1): 10-19.

9. Jimenez- Diez O, Ojeda-López RN. Estudiantes universitarios y el estilo de vida. Rev Iberoamerican de Porducción Academica y Gestión Educativa. 2017; 4(8). http://www.pag.org.mx/index.php/PAG/ article/view/723/1009.
10. Puerta K, De La Rosa- Luna R, Ramos A. Niveles de actividad física y su relación entre la distribución por sexo y programa académico en una universidad. MHSalud. 2019; 16 (2): 1-10. doi: https:// doi. org/10.15359/mhs.16-2.4.

11. Alvarez-Condo G, Guadalupe-Vargas M, MoralesMurillo H, Robles-Amaya J. El sedentarismo y la actividad física en trabajadores administrativos del sector público. Rev Ciencia UNEMI. 2016; 9(21): 116-124. http://ojs.unemi.edu.ec/index.php/ cienciaunemi/article/view/399/329.

12. Mahecha-Matsudo S. Recomendaciones de actividad física: un mensaje para el profesional de la salud. Rev Nutr Clin Met. 2019; 2(2): 44-54. doi: https://doi.org/10.35454/rncm.v2n2.006.

13. Lamotte M. Factores de riesgo cardiovascular y actividad física. EMC- Kinesiterapia. 2016; 37(2): 1-7. doi: https://doi.org/10.1016/ S12932965(16)77465-2.

14. Organización Mundial de la Salud. Versión Panamericana del Instrumento STEPS (principal y ampliada). 2010. http://www.who.int/ncds/ surveillance/steps/instrument/PAHO_STEPS Instrument_v2.1_ES.pdf.

15. Stewart A, Marfell-Jones M, Olds T, De Ridder H. Protocolo Internacional para la Valoración Antropométrica ISAK. 2011: 1-126.

16. The Helsinki Declaration Orvosi Hetilap. 1965; 106: 1715-1716.

17. Morales G, Del Valle C, Soto Á, Ivanovic D. Factores de riesgo cardiovascular en estudiantes universitarios Cardiovascular risk factors of college students. Nut Rev Chil. 2013; 40(4): 391-396. doi: http://dx.doi. org/10.4067/S0717-75182013000400010.

18. Fares J, Al-Hayek S, Jaafar J, Djabrayan N, Farhat A. Factors affecting body composition of Lebanese university students. Nutr Food Sci. 2018; 48(2): 228-243. doi: https://doi.org/10.1108/NFS-082017-0172.

19. Lotrean LM, Stan O, Lencu C, Laza V. Dietary patterns, physical activity, body mass index, weightrelated behaviours and their interrelationship among Romanian university students-trends from 2003 to 2016. Nutr Hosp. 2018; 35(2): 375-383. doi: 10.20960/nh.1296.

20. Nkeh-Chungag B, Mxhosa T, Mgoduka P. Association of waist and hip circumferences with the presence of hypertension and pre- hypertension in young South African adults. Afr Health Sci. 2015; 15(3): 908-916. doi: http://dx.doi.org/10.4314/ahs. v15i3.27. 
21. Porto-Arias J, Lorenzo T, Lamas A, Regal P, Cardelle-Cobas A, Cepeda A. Food patterns and nutritional assessment in Galician university students. J Physiol Biochem. 2018. 74(1): 199-126. doi: https://doi.org/10.1007/s13105-017-0582-0.

22. Conde M, Tercedor P. La actividad física, la educación física y la condición física pueden estar relacionadas con el rendimiento académico y cognitivo en jóvenes . Revisión sistemática. Arch Med Deport. 2015; 32(2): 100-109.

23. Rodriguez F, Palma X, Romo Á, Escobar D, Aragu B, Espinoza L, et al. Hábitos alimentarios , actividad física y nivel socioeconómico en estudiantes universitarios de Chile. Nutr Hosp. 2013; 28(2): 447-455. doi: http://dx.doi.org/10.3305/ nh.2013.28.2.6230.

24. Moreno-Arrebola R, Fernández-Revelles A, LinaresManrique M, Espejo-Garcés T. Revisión sistemática sobre hábitos de actividad física en estudiantes universitarios. Sportis. 2018; 4(1): 162-183.

25. Castañeda C, Zagalaz M, Arufe V, Campos-Mesa M. Motivos hacia la práctica de actividad física de los estudaintes universitarios Sevillanos. Rev Iber Psicol Ejerc Deport. 2018; 13(1): 79-89.

26. Concha-Cisternas Y, Guzmán-Muñoz E, ValdésBadilla P, Lira-Cea C, Peterman F, Celis-Morales C. Factores de riesgo asociados a bajo nivel de actividad fisica y exceso de peso corporal en estudiantes universitarios. Rev Med Chile. 2018; 146: 840-849. doi: https://doi.org/10.4067/s003498872018000800840.

27. Morales G, Balboa-Castillo T, Muñoz S, Belmar C, Soto Á, Schifferli I, et al. Asociacion entre factores de riesgo cardiometabólicos, actividad física y sedentarismo en universitarios chilenos. Nutr Hosp. 2017; 34(6): 1345-1352. doi: https://doi. org/10.20960/nh.1060.

28. Castañeda-Vasquez C, Campos-Mesa M, Del Castillo O. Actividad física y percepción de salud de los estudiantes universitarios. Rev Fac Med. 2016; 64(2): 277-284. doi: http://dx.doi.org/10.15446/ revfacmed.v64n2.53068.

29. Deliens T, Deforche B, De Bourdeaudhuji I, Clarys P. Determinants of physical activity and sedentary behaviour in university students: a qualitative study using focus group discussions. BMC Public Health. 2015; 15: 201. doi: https://doi.org/10.1186/s12889015-1553-4.

30. Maphisa J, Young C. Addictive Behaviors Risk of alcohol use disorder among South African university students : The role of drinking motives. Addict Behav. 2018; 82: 44-49. doi: https://doi. org/10.1016/j.addbeh.2018.02.016.
31. Atwell K, Abraham C, Duka T. A parsimonious, integrative model of key psychological correlates of uk university students alcohol consumption. Alcohol Alcohol. 2011; 46(3): 253-260. doi: 10.1093/alcalc/ agr016.

32. Tembo C, Burns S, Kalembo F. The association between levels of alcohol consumption and mental health problems and academic performance among young university students. PLoS One. 2017; 12(6): 1-13. doi: http://dx.doi.org/10.1371/journal. pone. 0178142 .

33. Peltzer K, Pengpid S. Concurrent tobacco use and binge drinking among university students in 30 countries in Africa, Asia, Latin America and the Caribbean. Int J Ment Heal Addict. 2018; 16: 164-174.

34. Saridi M, Nanou A, Vasilopoulos C, Kourakos M, Skliros E, Toska A, et al. Smoking habits among greek university students after the financial crisis. Asian Pac J Cancer Prev. 2017; 18(5): 1329-1335. doi: 10.22034/APJCP.2017.18.5.1329.

35. Pi R, Vidal P, Brassesco B, Viola L, Aballay L. Estado nutricional en estudiantes universitarios: su relación con el número de ingestas alimentarias diarias y el consumo de macronutrientes. Nutr Hosp. 2015; 31(4): 1748-1757. doi: http://dx.doi. org/10.3305/nh.2015.31.4.8399.

36. Sprake E, Russell J, Cecil J, Cooper R, Grabowski P, Pourshahidi L, et al. Dietary patterns of university students in the UK: a cross-sectional study. Nutrit J. 2018;19:90. doi: http://dx.doi.org/10.1186/s12937018-0398-y.

37. Valladares M, Durán E, Matheus A, Durán-Aguero S, Obregón A, Ramírez-Tagle R. Association between eating behavior and academic performance in university students. J Am Coll Nutr. 2016; 35(8): 699-703. doi: 10.1080/07315724.2016.1157526.

38. Sanchez V, Aguilar A, Gonzalez F, Esquius L, Vaqué C. Evolución en los conocimientos sobre alimentación: una intervención educativa en estudiantes universitarios. Rev Chil Nutr. 2017; 44(1): 19-27. doi: http://dx.doi.org/10.4067/S071775182017000100003 . 